\title{
Exploring the Reasons for the Inadequate Clinical Competency in the Newly Graduated Nurses: A Qualitative Study
}

\author{
Research Article
}

\section{Reza Negarandeh ${ }^{1}$, Hadi Ahmadi Chenari², Parvin Mahmoodi ${ }^{3 *}$}

\author{
1. PhD, Professor, Nursing and Midwifery Care Research Center, School of Nursing and Midwifery, Tehran \\ University of Medical Sciences, Tehran, Iran. \\ 2. PhD, Assistant Professor, Department of Medical Surgical Nursing, \\ Ferdows School of Paramedical and Health, Birjand University of Medical Sciences, Birjand, Iran. \\ 3. PhD Candidate, School of Nursing and Midwifery, Tehran University of Medical Sciences, Tehran, Iran.
}

\begin{abstract}
Aim: The most important mission of Healthcare systems is to deliver safe, efficient, and high-quality patient care. Manpower is the key pillar in achieving this goal. Studies show that newly graduated nurses do not have sufficient clinical competence to care for patients. This study aimed to explore the reasons for inadequate competence of newly graduated nurses. Method: The present study is a qualitative study. Participants in this study were 30 individuals, including nursing students, newly graduates nurses, nursing faculties, clinical nurses, nursing managers and deputies for the education of nursing schools who were selected by purposeful sampling method. Data were collected through semi-structured interviews. The data collection continued to data saturation. Data were analyzed using qualitative content analysis in MAXQDA 10 software. Results: Three main categories emerged as reasons for the inadequate clinical competency in the newly graduated nurses i.e. inefficient mechanism of student recruitment, ineffective education, and gloomy outlook for the nursing profession. Conclusion: Training qualified clinical nurses is a dynamic process that requires enrolling the appropriate individuals, preparing a suitable infrastructure to train, training them effectively, providing in-service training, and providing the necessary motivation for professional development in the health care settings.
\end{abstract}

Key Words: Clinical competence, Newly graduate nurses.

\section{Introduction}

Over 19 million nurses care for individuals, families, and communities worldwide every day (1). Nurses are working in a variety of settings such as hospitals, physicians' offices, outpatient care facilities, clinics, nursing homes, schools, and community health centers, but the majority of nurses $(61 \%)$ are employed at state, local and private hospitals (2). More than 10\% of nursing staff in each hospital are novice nurses which are on the rise due to the increasing number of nursing graduates entering the health care settings (3). Studies have shown that newly graduated nurses are not clinically competence in caring for patients (4). As nurses represent the largest number of healthcare providers and have the widest contact with the population, their readiness to offer quality care is central to safeguard clients (2). Observational studies associated increased mortality of patients with lower professional competence of nurses (3).

* Corresponding Author:

Parvin Mahmoodi

$\mathrm{PhD}$ Candidate,

Postgraduate Department, School of Nursing and Midwifery, Tehran University of Medical Sciences, Tohid Square, Nosrat St, Tehran, Iran.

Email Id: Mahmoodi.parvin@gmail.com
The concept of clinical competence has been taken seriously in recent years to protect people and a new generation of competency-based curriculum has been developed (2). But despite these efforts review of literature has shown that the definition of competence remains ambiguous and inconsistent, and its relation to terms such as capability, expertise, and performance is unclear $(3,5)$. Benner et al.1982 has defined nurse competence as the ability to perform a task with a desirable outcome under various conditions of the real world (6). The concept of competence is reflected in the knowledge, critical thinking, technical and interpersonal skills that a professional person fits into a particular situation (7).

Lee-Hsiehet al. 2003 proposed a definition of clinical nursing competence that comprises four dimensions: 1) Caring competence, that encompasses nursing process; 2) Communication/Coordination competence with clients, families, and colleagues; 3) Management/Teaching competence, which includes infection control, cost management, and patient education skills; and 4) Self-growth competence, concerns the acceptance of criticism, displaying caring behavior, sharing knowledge, and nursing ethics. This means that nursing practice requires a sophisticated combination of knowledge, skills, values and attitudes. Therefore competency is acquired through the acquisition of a number of abilities (8). 
The results of quantitative studies show that newly graduated nurses from universities around the world do not have the ability, competence, and confidence to provide quality care when entering the nursing workforce $(4,9-12)$. In a number of studies, both newly graduated and senior nurses believed that newly graduated nurses did not feel competent to perform tasks (13). A systematic review of 13 quantitative studies showed that newly graduated nurses were not clinically competence to enter the patient's bedside (11). Kavanagh et al. 2017 found that the reason for the unpreparedness of graduates for work in complex environments is the lack of deep learning in academic nursing programs. Graduates in the first trimester of employment have the most anxiety and abandonment of work due to their low level of competence (10).

Qualitative studies have also clarified the experiences of newly graduated nurses entering the nursing workforce $(4,13)$. These studies concluded that newly graduated nurses experience stress, job dissatisfaction, frustration, and shock when entering the patient's bedside. In the UK, where students are educated for 2,300 hours in different practicum environments, students lack confidence and felt the need for further training in their curriculum (14). A number of qualitative studies have also examined the causes of stress and shock experienced by newly graduated nurses when entering the nursing workforce. Some of these studies obscure the expected roles of the nurses(15), some of them theory-practice gap which means not all of the theories taught at the university are applicable in practice (16) and some also hold that education system is responsible for graduating inadequate nurses $(17,18)$. But the reasons for the inadequate clinical competency in the newly graduated are not clear. Therefore, this study aimed to explore the reasons for the inadequate competence of newly graduated nurses.

\section{Method}

\section{Study Design and Participants}

In this qualitative study, 30 participants were studied. Participants were selected from students, newly graduates, nursing instructors, nursing faculties, clinical nurses, nursing managers (matrons, supervisors, and head nurses) and deputies for the education of nursing schools (Table 1). Purposeful sampling was used with the maximum variation of age, gender, clinical work experience, and educational level.

Table 1: Demographic profile of the participants $(n=30)$

\begin{tabular}{|c|c|c|c|c|}
\hline Job experience (years) & Job & Age (years) & Gender & Participant \\
\hline 18 & Faculty member & 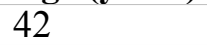 & Female & P1 \\
\hline 10 & Nurse Faculty & 38 & Male & P2 \\
\hline - & Nursing student & 22 & Female & P3 \\
\hline 20 & Faculty member & 48 & Female & P4 \\
\hline- & Graduated nursing student & 23 & Female & P5 \\
\hline 7 & Clinical Nurse & 30 & Male & P6 \\
\hline 5 & Faculty member & 32 & Male & P7 \\
\hline 22 & Nurse supervisor & 38 & Female & P8 \\
\hline 11 & Faculty member & 35 & Male & P9 \\
\hline 12 & Faculty member & 38 & Female & P10 \\
\hline 28 & Head Nurse & 51 & Male & P11 \\
\hline 18 & Nurse Supervisor & 40 & Male & P12 \\
\hline 10 & Faculty member & 34 & Male & P13 \\
\hline 5 & $\mathrm{PhD}$ candidate of nursing & 28 & Female & P14 \\
\hline 5 & Faculty member & 30 & Female & P15 \\
\hline 25 & Metron & 48 & Female & P16 \\
\hline 18 & Faculty member & 42 & Female & P17 \\
\hline 17 & Deputies for the education & 40 & Male & P18 \\
\hline 10 & Faculty member & 35 & Male & P19 \\
\hline - & Nursing student & 21 & Female & P20 \\
\hline 6 & PhD Student of nursing & 29 & Male & P21 \\
\hline 7 & Faculty member & 31 & Male & P22 \\
\hline 2 & Nurse & 24 & Female & $\mathrm{P} 23$ \\
\hline 10 & Nurse & 32 & Female & P24 \\
\hline 15 & Faculty member & 39 & Female & $\mathrm{P} 25$ \\
\hline 17 & Faculty member & 41 & Male & P26 \\
\hline 9 & Faculty member & 38 & Male & P27 \\
\hline 4 & $\mathrm{PhD}$ candidate of nursing & 30 & Male & P28 \\
\hline- & Nursing student & 22 & Female & P29 \\
\hline 12 & Faculty member & 38 & Male & P30 \\
\hline
\end{tabular}

\section{Data Collection}

Data were collected through semi-structured interviews using the interview guide. All interviews were conducted individually. The interviews began with an open-ended and general question about the research topic;for example, "What skills and competencies are 
necessary for nursing graduates?" Other questions include: "What are the reasons for the inadequate competency of graduates?", and "What factors hinder sufficient clinical competence in graduates?" During the interview, based on the interviewer's answers the probing questions such as "what do you mean?", "Can you explain more?", "why", and "how" was used to examine, clarify the participants' experiences.

The time and location of the interview sessions were determined by agreement between the interviewee and the researcher. Interviews were conducted by an interviewer. Therefore, the interviews were conducted in a quiet environment inside the hospital or university to maintain the participants' peace and concentration. Each interview lasted 45 minutes on average. Interviews were recorded using a digital device with the consent of participants. Participants were told that the audio recording would be held whenever they want. The interviews were continued until data saturation, where the researcher begins to hear the same comments again and again and no new theme or idea emerged (19).

\section{Data analysis}

Data were analyzed using a content analysis approach. The researcher first transcribed the interviews verbatim from the recorded file and read repeatedly to achieve a sense of the whole. Then, the analysis was conducted word by word, line by line or paragraph by paragraph and the data were broken down into semantic units and labeled by an invivo or inferential code. After specifying open codes, the codes were grouped according to similarities and differences in categories. Subcategories was grouped with similar meanings and considered as a category and categories are grouped as main categories (20). MAXQDA 10 software was used for data management. Interviews were conducted in Farsi.

\section{Trustworthiness}

This study used the proposed criteria of Guba and Lincoln to evaluate the validity of the data (21). The prolonged engagement with the participants during the data gathering period helped to build trust and a better understanding. The research team checked the interview data and findings at each step of the study. In addition, interpretations and conclusions were checked with the members. Peer debriefing conducted to indicate our position toward data and analysis.

\section{Ethical considerations}

The Ethics Committee of Tehran University of Medical Sciences reviewed and approved the study protocol. Ethical considerations, such as maintaining confidentiality; explaining about the importance, objectives of study for participants; and getting written consent for being participants in the research as well, recording the interviews were observed.

\section{Findings}

This study determined the causes of the inadequate clinical competence of newly graduated nursing staffs as three main categories of inefficient mechanism of student recruitment, ineffective education, and gloomy outlook for the nursing profession. in detail.

Here, we will discuss each of these categories

\section{Ineffective mechanism of the student recruitment}

The nursing field has been known as one of the hardest professions. A person who intends to enter the nursing field must have some characteristics such as proper physical and psychological capabilities to act as a nurse. In Iran, people participate in a university entrance exam known as "Konkor" and then, they can choose a field of study according to their score if they pass the exam. Thus, other physical and psychological factors, and interest of student did not consider carefully. This category has two subcategories:

\section{Nursing students' lack of interest in the study field}

In general, interest is one of the essential components to choose afield, in particular, in the field of nursing, as it is directly related to human life. Unfortunately, some students enter the field of nursing, not out of their interest, but as a compulsion. In Iran, some popular fields such as dentistry, medicine, and pharmacy enjoy both high social status and income. As a result, the entrance exam candidates are more interested to choose these fields. It is clear that those who do not obtain the required score will inevitably enter the nursing field. "Nursing students in the Iranian education system are mainly forced to choose the nursing field because of it's good market and income, or due to non-admission in the fields of medicine and dentistry at some universities in Tehran," one of the associate professors of nursing said.(P15).

Another nursing Ph.D. candidate said: "Recruitment of a nursing applicant is mainly based on the obtained score of the entrance examination, not the interest that is the most effective factor in this matter. Students' recruitment is required to be based on interviews, scientific exams and psychology tests like personality and interests.

\section{Lack of paying attention to the physical, psychological, and mental capacities of students admitted in the nursing}

According to the participants' interviews, entering the nursing field is not acceptable without considering the personality, desire, intrinsic motivations, and even ignoring students' physical and psychological health.

To explain this idea, one of the nurse faculty said: "Every year, in the nursing field, about one to two admitted students do not have both physical and psychological health. So how this student intends to learn the necessary skills as well as to take care of the patient? Can a nursing student with the history of hospitalizing for mental disease or showing mental disorder signs be a good caregiver? Can a very shortsighted student be able to demonstrate good nursing care skills even if they are well educated?"(P2) 
Ineffective education

Ineffective education was another reason that participants mainly point out to it as the cause of low competence of newly graduated nurse. In general, this category includes five subcategories as follows: weaknesses of curriculum, improper practicum implementation, the gap between theoretical and clinical education, and the lack of standard infrastructures and educational environment.

\section{Weaknesses of Curriculum}

This subcategory denotes the lack of both consistency and appropriateness of both theoretical and practical courses. This means that there is no equilibrium between the number of theoretical courses and practicum, based on which there is a long gap between their teaching times in some cases.

The main weaknesses of the curriculum also point out to the incompatibility of the syllabus with the actual needs of the Iranian nurses. A participant who head nurse for about 15 years says: "In my opinion, the problem of curriculum is the mismatch with the needs of the community, and the compulsory specialty courses are often decreased (theoretical and clinical), without investigating and identifying the needs, problems, and shortcomings of both students and graduates".(P18)

Another weakness of the nursing curriculum is the lack of paying attention to comprehensive patient care, in which the student only learns mechanical care whereas he/she does not pay attention to the patients' cultural differences in care. This can be concluded from the explanations of one of the nursing professors: "in the curriculum, several skills such as paying attention to the patient privacy, respect, cultural competence, and teamwork and interdisciplinary work, are mentioned superficially; so the students are often not familiar with such concepts".(P4)

\section{Implementing inappropriate internships}

In fact, implementing the inappropriate internships means paying a little attention to evidencebased care, the implementation of the nursing process, designing and the using course plans for the practicum. An undergraduate nursing student who was studying in seventh-semester said: "students are not taught how to use and learn the important content from the evidencebased sites; so the student does not learn evidencebased care, and her/his knowledge is exclusively obtained from worthless Persian sites that often provide inaccurate content ".(P3)

\section{The gap between theoretical and clinical education}

The gap between theory and practice education refers to the island's performance of the various nursing pillars, including education, practice, research, and leadership. This fragmentation impedes the clinical nurses to collaborate with each other to educate the next generation of clinical nurses. On the other hand, nursing professors do not have adequate control over the healthcare systems. As a result, the gap between education and health care staff would result in the disruption of nursing students' education. These issues can be concluded from the descriptions of a matron. "If colleges collaborate with hospitals and then employ some skilled nursing staff in both theoretical and clinical education, then nursing department and as a result, the nurses will be involved in the students' clinical education. If faculty members collaborate more with clinical staff, this may result in eliminating the gap between practice and education and decreasing the students' educational problems, so competencies are learned as well."(P16)

\section{The lack of standard infrastructures and educational environment}

One of the essential elements for nursing education is providing the suitable educational infrastructure. In recent years, the small towns have been allowed to establish a nursing school without having proper educational field and staff. The lack of educational facilities due to economic sanctions, the lack of standard clinical wards, overcrowded classrooms, and the inappropriate hospital environment for education indicate that the educational environment is really not standard. Thus, it is not possible for students to experience all clinical skills due to the limited variety of patients, learning situations, devices, and a large number of students. "The hospitals are only nominally educational, but the basic principles of the educational hospital are not observed, In addition, the standard clinical departments and professional nurses are seen less likely to be found as a role model for students. " said one professor. (P2)

\section{Inefficient instructors/professors}

The role-models have a significant influence on internalizing a nurse's personality in nursing students. This role-model can be either good or bad, or either an educator or nursing practitioner. Unfortunately, some educators lack the professional qualifications and confidence. They do not provide enough motivation for students as well as do not utilize new teaching methods, avoid working in practice and are only thinking about promotion. Meanwhile, they are only thinking about promotion. "It is worthwhile to mention that in the last few years, we have unfortunately been experiencing a decline in the number of experienced professors in educational settings. Today, the academic centers often employ new graduates with no training or clinical experience for many courses, especially in the clinical education; even they have not sufficient experience or knowledge or attitude they needed", a supervisor said. (P8)

\section{Gloomy outlook for the nursing profession}

This concept denotes the unfavorable nursing conditions and the disappointment of positive changes in the profession.

\section{Unfavorable Nursing Conditions}

Working day and night shifts in holidays is among critical factors that create some unfavorable conditions for the nursing field. In this regard, the night work may disrupt the biological clock of the nurses and 
interfere with the individual's duties and roles in the family. Although the nursing field is a highresponsibility profession and is associated with high occupational stress, nurses' income is low. There is also a lot of discrimination in the workplace between the nurse and the physician. Sometimes, the nurses are humiliated by doctors. One of the participants said: "Nurses are not able to utilize the competency acquired during the undergraduate education after entering the healthcare settings. They used to work shifts with the least use of these competencies. This challenge is mainly due to the workforce shortages, as well as long overtime, and working at night shifts".

\section{The Disappointment of Positive Changes in the profession}

In recent decades, the nurses' efforts have led to forming several professional organizations, based on which the Iranian nurses have sought to enhance the status of the nursing profession. Nevertheless, a small proportion of nurses are members of these organizations. This reflects the nurses' disappointment with the positive changes in a profession (22). The nursing conditions including workload and low salaries deprive nurses of the necessary motivation for professional development. "Nurses forget about all their professional behaviors, except medication and following the doctor's orders, due to high workload, low salary, and excessive shifts" said one nurse.(P19)

\section{Discussion}

This study identified some main reasons for the inadequate clinical competence of newly graduated nurses. In this regard, the ineffective student recruitment mechanism is one of the most important reasons. The results of this study revealed that student's lack of interest in nursing, and a lack of attention to the physical, psychological and mental capacities of a nursing student are a sign of an inappropriate recruitment process.

The recent studies show that the quality of nursing care and the practice of nurses are significantly influenced by the adherence to professional values (23). Today, the core values of nursing consist of: altruism, equality, freedom, respect for human dignity, justice, honesty, satisfaction, in the individuality and unity of values (24).

The relationship between personality characteristics and nurses' professional values is assessed in a study which it's results showed that if the extroversion, friendliness, and conscientiousness dimension of nurses are stronger, the better care for others is provided (25).Choosing the appropriate persons for nursing noticeably increases the probability of training competence and successful nurses. This is accomplished by changing the nurses' recruitment mechanism, incorporating personality and interest tests into the selection process.

Ineffective education was another reason mentioned by the participants, which implies some deficiencies in the levels of infrastructure, the curriculum, instructors, and practicum. The nursing education curriculum contains some weaknesses such as the lack of consistency and proportionality between both theory and practice, a lack of turning shifts in the student curriculum, and not being up-to-date. The study by Al Awaisi et al. 2015 showed that Omani graduates consider the gap between theory and practice to be the cause of the shock experienced by them during the transition period (9). This reveals that when they enter the workplace, they encounter some limited practical experience, but they have extensive theoretical knowledge that can be difficult to practice. Another study emphasizes the need for coordination between what is being taught and what is being done in the practice, and they believe that due to the weakness of basic science courses such as physiology, anatomy, and pharmacology and the harsh nature of nursing education, nursing students after entering the hospital and going to the patient's bedside are not well-prepared for applying the lessons taught in the practice (26).

The results of this study showed that sometimes, effective and experienced professors are not employed for clinical education. On the other hand, hospitals and colleges encounter a lack of the necessary training facilities, a variety of patient departments and patient cases for education. Thus, all of these factors would prevent the student to fulfill the required qualifications. At the same time, Nazari and Mohammadi emphasized the competence of clinical educators and their mastery of high-level knowledge, and skills to attain professional competence as a necessity condition (27). Another research demonstrated that effective training in the process of acquiring clinical skills, recruiting and employing experienced clinical educators could affect the development of collaboration among educators to enhance the educational environment and clinical departments. As such, some efforts to improve the educational environment may have a significant impact (26).

Moreover, the presence of a qualified educator who can evaluate the students' clinical practice can play a critical role-model for the students as well as for transforming the qualified, competent, and professional nurse thorough the explicit and hidden curriculum. Some concepts such as patient privacy, respect, cultural competence, teamwork and interdisciplinary should be well internalized in students. The evidence suggests that effective clinical supervision can significantly increase students' motivation and enhance their professional identity. Furthermore, it strengthens the ability of communication between knowledge and practice, develops a sense of support for them, and promotes their attitudes towards their profession (27). It should be noted that supportive clinical environment could significantly improve both students' motivation and professional practice.

Here, the issue of clinical education and preparing students to accept different roles in the health services is one of the most important activities and goals of nursing schools, which can be possible with the help of the effective professors and the necessary tools (28). One of the essentials of appropriate training is the 
suitability of the physical environment, facilities, professors and personnel. In other words, the environment that has good collaboration between individuals may lead to achieving an effective education (29). Besides, in this regard, the results of some studies indicate that there is no proportion between the facilities and requirements for student practice, and it has not enough capacity for the number of students (30).

The results of the current research confirmed that the practicums are held on a superficial and nonprogrammatic basis, so there is no sense of commitment and responsibility among the students In this regard, some scholars believe that offering a practicum course plan at the beginning of clinical education will improve student satisfaction, understanding of professional responsibilities, expectations, and tasks, better clinical skills learning, and application of theoretical lessons in practice (18). It is worthwhile to note that the practicum course plan contains some important items such as apprenticeship behavioral goals, department regulations, student and professor task descriptions, how and when to evaluate students, and the resources needed by students Furthermore, to get ready for work, the students require to be trained in learning environments with new techniques and equipment to get ready for work.

In this way, gloomy outlook for the nursing profession is another reason pointed out to by the participants, which denoted difficult nursing conditions and the disappointment of positive changes in the profession. For this reason, nurses do not believe in professional promotion and consequently, they should have followed the vocational approaches. Discrimination between nurse and physician, humiliation by the physician, and low social nurse status are other results of the current study. This kind of attitude has now existed in Iran for many years (31). Nursing contains some hierarchical physician-nurse relationships that affect them to feel underwhelmed, frustrated, and unable to make clinical decisions. This is because they are expected to follow the physician's instructions without any delay $(5,32)$

\section{Conclusion}

This study attempted to explore the main causes of the inadequate clinical competence of the newly graduated nurses. It can be observed that training a qualified nurse requires choosing the appropriate individuals and preparing a suitable infrastructure to train and improve the prospects of the profession. In this regard, it is suggested to provide some appropriate infrastructure, including colleges with appropriate educational aids and hospitals with a variety of training facilities as diversified learning opportunities, and holding regular practicum (using evidence-based care and nursing process). Furthermore, the curriculum should be revised and modified periodically. The respect-based communication within the healthcare system and community, employing competent and efficient instructors, providing sufficient nursing staff and motivation for staff to improve themselves are also suggested as well. Therefore, removing the barriers to train competent nurse requires improving the cultural, educational, and professional context.

It is recommended that hospitals consider programs that include strategies for clear communication and conflict management, prioritization skills, leadership development, critical thinking, clinical reasoning and simulation scenarios to enhance the clinical competence of graduates appropriate for their work environment.

\section{Acknowledgements}

The authors would like to thank all parents who participated in this study and so sincerely shared their experiences. This study has been funded and supported by Tehran University of medical sciences (TUMS); grant no. 37170 .

\section{Conflict of interests} authors.

No conflict of interest has been declared by the

\section{References}

1. Organization WH. Enhancing nursing and midwifery capacity to contribute to the prevention, treatment, and management of noncommunicable diseases. Human Resources for Health Observer. 2012;12:32.

2. Beogo I, Rojas BM, Gagnon M-P, Liu C-Y. Psychometric evaluation of the French version of the Clinical Nursing Competence Questionnaire (CNCQ-22): A cross-sectional study in nursing education in Burkina Faso. Nurse education today. 2016;45:173-8.

3. Berkow S, Virkstis K, Stewart J, L C. Assessing new graduate nurse performance. Nurse educator. 2009;34(1):17-22.

4. Hsu L-L, Hsieh S-I. Development and psychometric evaluation of the competency inventory for nursing students: A learning outcome perspective. Nurse Education Today. 2013;33(5):492-7..

5. Applin H, Williams B, Day R, Buro K. A comparison of competencies between problembased learning and non-problem-based graduate nurses. Nurse Education Today. 2011;31(2):129-34.

6. Tilden VP, TildenS. Benner, P.(1984). From novice to expert, excellence and power in clinical nursing practice. Menlo Park, CA: Addison-Wesley Publishing Company, 307 pp., \$12.95 (soft cover). Research in Nursing \& Health. 1985;8(1):95-7.

7. Davoudi M, Mohammadi Y, Yazdanparast E, Ahmadi Chenari H, Eslami H, Rajabi R et al . The effect of Multimedia Method of Education on Weight loss in Hemodialysis Patients. IJN. 2016; 28 (98) :31-38.

8. Shureshi, P., Ahmadi Chenari, H., Ahmadi, M., Jesmi, A. A. (2015). Effect of education by lecture and pamphlet methods on soldiers knowledge about meningitis disease. Journal of Military Medicine, 17(3), 181-186

9. Al Awaisi H, Cooke H, Pryjmachuk S. The experiences of newlygraduated nurses during their 
first year of practice in the Sultanate of Oman-A case study. International journal of nursing studies. 2015;52(11):1723-34.

10. Kavanagh JM, Szweda C. A crisis in competency: The strategic and ethical imperative to assessing new graduate nurses' clinical reasoning. Nursing Education Perspectives. 2017;38(2):57-62.

11. Pasila K, Elo S, Kääriäinen M. Newly graduated nurses' orientation experiences: A systematic review of qualitative studies. International Journal of Nursing Studies. 2017;71:17-27.

12. Dlamini CP, Mtshali NG, Dlamini CH, Mahanya S, Shabangu T, Tsabedze Z. New graduates' readiness for practice in Swaziland: An exploration of stakeholders' perspectives. Journal of Nursing Education and Practice. 2014;4(5):148.

13. Ahmadi Chenari H, Zakerimoghadam M, Baumann SL. Nursing in Iran: Issues and Challenges. Nurs Sci Q. 2020 Jul; 33(3):264-267.

14. Bradshaw A, Merriman C. Nursing competence 10 years on: fit for practice and purpose yet? Journal of Clinical Nursing. 2008;17(10):1263-9.

15. Häggman-Laitila A, Elina E, Riitta M, Kirsi S, Leena R. Nursing students in clinical practicedeveloping a model for clinical supervision. Nurse Education in Practice. 2007;7(6):381-91.

16. SANAGOO A, NOMALI M, JOUYBARI L. Explanation of educational equity among Medical Sciences students: evaluation of Medical Sciences students opinions and experiences. 2011.

17. Fatemi S, Moosavi S, Nikro R, Mohemkarkherandish S. Exploration of Medical Sciences Students and Educational Custodians View about Educational Equity in Clinical Environment. Research In Medical Education. 2017;8(4):1-10.

18. Heshmati NF, Vanaki Z. Effective clinical instructor: a qualitative study. 2009.

19. Saunders B, Sim J, Kingstone T, Baker S, Waterfield J, Bartlam B, et al. Saturation in qualitative research: exploring its conceptualization and operationalization. Quality \& quantity. 2018;52(4):1893-907.

20. Elo S, Kyngäs $H$. The qualitative content analysis process. Journal of advanced nursing. 2008;62(1):107-15.

21. Guba EG, Lincoln YS. Fourth generation evaluation: Sage; 1989.
22. Esmaeili M, Dehghan-Nayeri N, Negarandeh R. Factors impacting membership and nonmembership in nursing associations: A qualitative study. Nursing \& health sciences. 2013;15(3):265-72.

23. Shahriari M, Baloochestani E. Applying professional values: the perspective of nurses of Isfahan hospitals. Journal ofmedical ethics and history of medicine. 2014;7.

24. Rassin M. Nurses' professional and personal values. Nursing ethics. 2008;15(5):614-30.

25. AllahyariBayatiani F, Fayazi S, Jahani S, SakiMalehi A. The relationship between the personality characteristicsand the professional values among nurses affiliated to Ahwaz University of Medical Sciences in 2014. Journal of Rafsanjan University of Medical Sciences. 2015;14(5):367-78.

26. McLaughlin K, Moutray M, Muldoon OT. The role of personality and self-efficacy in the selection and retention of successful nursing students: a longitudinal study. Journal of Advanced Nursing. 2008;61(2):211-21.

27. Nazari R, Mohammadi E. Effective organizational factors on a clinical teacher competency a qualitative research. Education Strategies in Medical Sciences. 2012;4(4):165-70.

28. Hashemiparast M, Negarandeh R, Theofanidis D. Exploring the barriers of utilizing theoretical knowledge in clinical settings: A qualitative study. International journal of nursing sciences. 2019;6(4):399-405.

29. Ebrahimi H, Hassankhani H, Negarandeh R, Azizi A, Gillespie M. Barriers to support for new graduated nurses in clinical settings: A qualitative study. Nurse education today. 2016;37:184-8.

30. Ebrahimi H, Hassankhani H, Negarandeh R, Jeffrey $\mathrm{C}$, Azizi A. Violence against new graduated nurses in clinical settings: A qualitative study. Nursing ethics. 2017;24(6):704-15.

31. Setoodegan E, Gholamzadeh S, Rakhshan M, Peiravi H. Nurses' lived experiences of professional autonomy in Iran. International Journal of Nursing Sciences. 2019.

32. Shohani M. Strategies of Iranian nurses to overcome professional discrimination: An explorative qualitative study. Nursing ethics. 2019;26(1):235-47. 